DOI: https://doi.org/10.46296/yc.v4i7.0041

\title{
MODELOS DE GESTIÓN DE LA CALIDAD PARA ORGANIZACIONES EDUCATIVAS
}

\section{QUALITY MANAGEMENT MODELS FOR EDUCATIONAL ORGANIZATIONS}

\author{
Sánchez-Muñiz Juan Carlos ${ }^{1 *}$; Pinargote-Macías Eleticia Isabel ${ }^{2}$ \\ 1 Universidad Técnica de Manabí (UTM) - Instituto de Posgrado. Portoviejo, Ecuador. \\ ${ }^{2}$ Docente Investigador de la Universidad Técnica de Manabí (UTM). Portoviejo, Ecuador.
}

*Correo: jsanchez7317@utm.edu.ec

\begin{abstract}
Resumen
La calidad es una exigencia vigente a nivel mundial en el área de la educación, constituye un indicador fundamental puesto que las instituciones educativas están sujetas a proporcionar un servicio de excelencia, por tanto, necesitan establecer modelos de gestión que le permitan alcanzar dichos niveles. El objetivo del presente artículo es de analizar diferentes modelos de gestión de la calidad que se pueden implementar a la gerencia de organizaciones educativas para la mejora continua y satisfacción de sus partes interesadas. La metodología utilizada en este estudio corresponde al método descriptivo que permitió analizar cinco modelos de gestión de la calidad que orientan y direccionan a los establecimientos educativos sobre procesos de satisfacción y expectativas de los usuarios. Los resultados del presente estudio permitieron establecer que el modelo ISO 21001:2018 posee un enfoque que busca satisfacer al cliente y posibilita la obtención de niveles de eficacia y eficiencia en todas las dimensiones educativas. El análisis de los diferentes modelos de gestión de la calidad, dan pautas para modificar los paradigmas y entender la dinámica de interacción entre sus elementos; reflexionar en la necesidad de realizar una evaluación institucional a los procesos de forma heterogénea desde las prácticas recurrentes de apreciación, hasta las formas de gestión directiva a fin de alcanzar la satisfacción de los clientes internos y externos.
\end{abstract}

Palabras clave: Calidad, modelos de gestión, calidad educativa, instituciones educativas, satisfacción.

\begin{abstract}
The quality of services is a requirement in force worldwide; In the area of education, it constitutes a fundamental indicator since educational institutions are subject to providing a service of excellence, therefore, they need to establish management models that allow them to achieve these levels. The objective of this article is to analyze different quality management models that can be implemented in the management of educational organizations for the continuous improvement and satisfaction of its stakeholders. The methodology used in this study corresponds to the descriptive method that allowed the analysis of five quality management models that guide and direct educational establishments on user satisfaction and expectations processes. The results of this study allowed to establish that the ISO 21001: 2018 model has an approach that seeks to satisfy the client and makes it possible to obtain levels of effectiveness and efficiency on the educational dimensions. The analysis of the different quality management models provides guidelines for modifying the paradigms of seeing and understanding what happens in daily life; reflect on the need to carry out an institutional evaluation of processes in a heterogeneous way, from recurrent appraisal practices, to forms of directive management in order to achieve the satisfaction of internal and external clients.
\end{abstract}

Keywords: Quality, management models, educational quality, educational institutions, satisfaction.

Información del manuscrito:

Fecha de recepción: 20 de abril de 2020

Fecha de aceptación: 09 de julio de 2020

Fecha de publicación: 10 de julio de 2020 


\section{Introducción}

Ante los constantes cambios de orden gerencial, los procesos de gestión están obligados a adaptarse a nuevas exigencias del precepto mundial, sobre todo de aquellas organizaciones dedicadas a generar conocimiento, pues necesitan que el aprendizaje sea de excelencia, por ello deben basar sus procesos en conocimientos científicos que conduzcan a las instituciones hacia la generación de una cultura organizacional y deslindarse de las formas tradicionales de la administración de la educación, ya que el producto que diseñan y el servicio que brindan exigen calidad, así surge la necesidad de conocer modelos de gestión que permitan alcanzar niveles de excelencia con estrategias innovadoras.

Ropa, (2014) afirma que "la calidad se traduce en aprendizaje, educación de valores, aptitudes, habilidades del conocimiento tecnológico, técnico y científico" (p.53). Es decir, que la excelencia educativa se consigue de acuerdo a la eficiencia y eficacia de los diferentes procesos, por lo que es fundamental que los directivos cuenten con conocimientos de métodos de gestión de la calidad para administrar eficientemente dicho servicio en cada uno de los procesos donde interaccionan los estudiantes, docentes, personal administrativo y padres de familia. No obstante, la realidad dista de esta integración y los cambios que se logran son a menor escala.

En Latinoamérica, aún se presentan problemas en la calidad educativa, tal como lo explica, Radic, (2017) "el contraste social marca una diferencia de asistencia escolar a partir de indicadores anclados en el poder, provocando estrechamiento en la enseñanza" (p.31). Esto deja en evidencia que aparte de las brechas sociales existentes en un derecho tan necesario como la educación, se necesita de manera urgente que los líderes educativos cuenten con herramientas de gestión para administrar con eficiencia su gestión, para ello, es menester empoderarse de una buena gestión donde se tenga como eje central el mejoramiento de la calidad de los procesos, reflejada en una planificación integrada, un liderazgo participativo, el desarrollo de sus integrantes y la comunicación efectiva. 
Este planteamiento exige el conocimiento y aplicación de los modelos de gestión de la calidad que sean configurables al sector educativo a fin de proporcionar efectividad en cada uno de componentes de la gestión, tanto en el área administrativa, pedagógica, seguridad escolar y la convivencia.

En Ecuador, según, Sandoval, (2017) indica que "la gestión educativa es considerada prioritaria para el país, porque se tiene la confianza que solo en ella se puede conseguir el mejoramiento social y económico, pues se encuentra consagrada como un deber y un derecho Constitucional" (p. 19).

Desde esta perspectiva, pese que para el estado ecuatoriano la educación es un derecho irrenunciable de todos sus ciudadanos, la brecha de desigualdades es muy notoria, por ello se requiere fortalecer las habilidades de gestión para lograr el cumplimiento de los estándares de la calidad educativa que orienten $y$ apoyen el mejoramiento continuo de los actores encargados de liderar la gestión de aprendizajes, gestión escolar y desempeño profesional. Urge entonces, rediseñar el rol que asumen los directivos, afín de responder a las exigencias de la educación en estos tiempos de acelerados cambios científicos y tecnológicos.

A respecto, De la Rosa y Guzmán, (2016) sostienen "los gestores de la educación necesitan integrarse para que optimicen sus esfuerzos con la finalidad de elevar la calidad de la educación" (p.29). Esto esclarece la necesidad de que los directivos orienten a la institución hacia la mejora continua.

En este sentido, se requiere de una transformación organizacional que vincule todo el accionar de la dinámica educativa, como las funciones directivas, de docencia y administrativas, así como el sistema de interacción entre ellos, establecidos a través de la visión, misión, políticas y valores que identifican a la institución, ya que por lo general muchos profesionales no han sido formados para ser profesores, administradores 0 gestores de la educación, ocasionando incertidumbre frente a la necesidad de orientar los procesos de formación de los estudiantes, y demás miembros de la comunidad educativa. 
En este sentido, se puede aseverar que existe poco conocimiento de la importancia de aplicar instrumentos de gestión internacionales que plantean estrategias para el alcance de la calidad, cuyos requisitos son ajustables a todas las organizaciones, sin importar su tipo o tamaño. Por tanto, el presente artículo tiene como objetivo analizar diferentes modelos de gestión de la calidad y su efectividad de aplicación en las instituciones educativas.

La calidad en el servicio educativo exige operatividad sistemática y sistémica donde se integren como un todo, los componentes que configuran entidad educativa, los mismos que deben estar sujetos a monitoreo y seguimiento permanente. Rubia, (2016) sostiene que "evaluar la calidad de la educación no es tarea simplemente técnica, sino que implica clarificación y compromiso pedagógico, sociopolítico y filosófico (...) para identificar factores, criterios $y$ procesos evaluativos de sistematización e interpretación de resultados" (p.129).

Los criterios citados reivindican a la evaluación de los conocimientos como factor clave para mantener la mejora continua del proceso formativo, organizando $y$ gestionando las intervenciones desde sus niveles macro, meso y micro, por medio de modelos que apunten hacia una línea de investigación-acción, con capacidad para atender y satisfacer las necesidades del entorno inmediato y mediato.

Ortiz, (2018), señala que la gestión de la calidad educativa "requiere medir los resultados del aprendizaje para poner en el centro al alumno y lo que este aprende, en lugar del sistema y de lo que se enseña" (p.59). Criterio con el que se difiere completamente, porque la calidad de la educación se centra en el compromiso de parte de los gestores educativos y su posición frente al cambio.

Las formas en que se proporciona el aprendizaje a los estudiantes, es responsabilidad del líder gestionar y garantizar la capacitación del docente para que éste cumpla su rol eficientemente, y sea capaz de promover organización, el trabajo efectivo y la participación, así como la manifestación de su compromiso con la mejora de los resultados. 
Según, Goairín y Díaz (2016) indica que "son cuatro las dimensiones de la gestión de la calidad educativa: gestión pedagógica, gestión de convivencia y participación escolar, gestión administrativa y gestión de seguridad escolar" (p.12), la primera evalúa las formas en que el docente realiza los procesos de enseñanza, es decir, cómo asume el currículo y lo traduce en una planeación didáctica; la segunda abarca el ámbito de la cultura organizacional, conformada por directivos, el equipo docente, normas, instancias de decisión, actores y factores que están relacionados con la manera de hacer las cosas en la institución.

La tercera se enfoca en las políticas; subsistemas y las líneas de acción de cada una de las instancias administrativas, es decir, la generación de proyectos, la visión panorámica del hecho educativo, las interrelaciones entre todos los actores, la planificación, programación, regulación y de orientación.

La cuarta dimensión contiene las exigencias para la creación y funcionamiento de las instituciones educativas, así como la prevención de riesgos psicosociales mediante la difusión educativa de las rutas y protocolos de actuación en situaciones de vulneración de derechos de niños, niñas y adolescentes que debe ser conocida por los miembros de la comunidad educativa, tal como se evidencia en la siguiente figura:

Figura 1. Dimensiones de gestión de calidad educativa

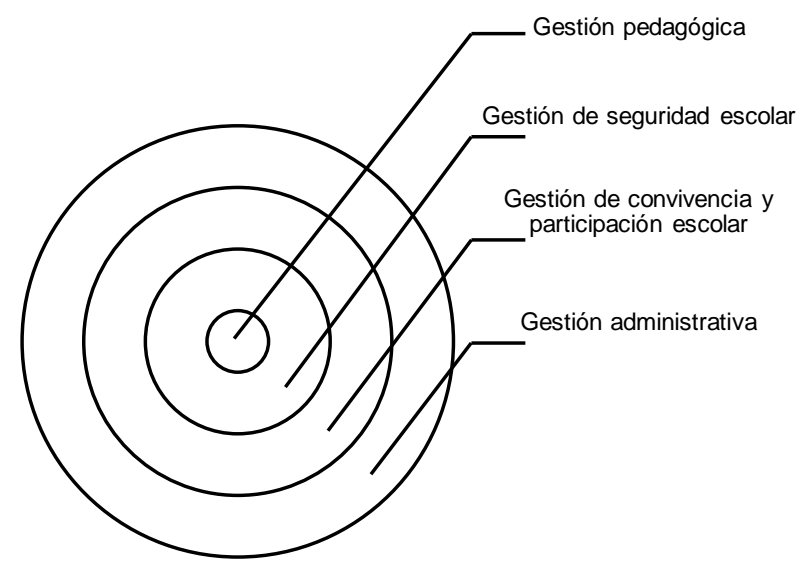

Fuente: Goairín y Díaz (2016)

Elaborado por: Autores

\section{Modelos de gestión de la calidad para organizaciones educativas}

Según, Ardila et al., (2016) explican que existen modelos que ayudan a cumplir los principios básicos para que la implantación del sistema de calidad sea provechoso; entre los modelos más utilizados se presentan en la figura No. 2: 
Figura 2. Modelos de gestión para organizaciones educativas

\begin{tabular}{|c|c|c|c|c|}
\hline $\begin{array}{c}\text { Modelo de las 5 } \\
\text { S }\end{array}$ & $\begin{array}{c}\text { Mantenimiento } \\
\text { productivo total, } \\
\text { (TPM) }\end{array}$ & $\begin{array}{c}\text { Ciclo PDCA } \\
\text { (Plan, Do, } \\
\text { Check; Act) }\end{array}$ & $\begin{array}{c}\text { ISO } \\
21001: 2018\end{array}$ & $\begin{array}{c}\text { KAIZEN (Kai = } \\
\text { cambio y Zen = } \\
\text { bueno) }\end{array}$ \\
\hline
\end{tabular}

Fuente: Ardila et al., (2016)

Elaborado por: Autores

\section{Modelo de gestión de la calidad de las $5 \mathrm{~S}$}

Según, Reyes et al., (2017) explica que el modelo de las $5 \mathrm{~S}$ es muy elemental, por ello es el indicado para iniciar procesos de gestión de calidad en organizaciones y su nombre está dado por las iniciales de sus 5 etapas de desarrollo en japonés:

Figura 3. Etapas de la metodología de las $5 S$.

\begin{tabular}{|c|c|c|c|c|}
\hline $\begin{array}{c}\text { Separar } \\
\text { todo lo } \\
\text { innecesario } \\
\text { क } \\
\text { ळ eliminarlo. }\end{array}$ & 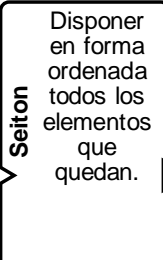 & $\begin{array}{c}\text { Mantener } \\
\text { limpias las } \\
\text { máquinas y } \\
\text { los } \\
\text { œ ambientes } \\
\text { de trabajo. }\end{array}$ & 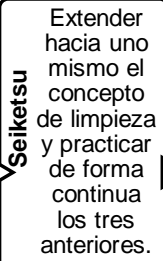 & $\begin{array}{c}\text { Construir } \\
\text { autodiscipli } \\
\text { na } \\
\text { na } \\
\text { mediante } \\
\text { के } \\
\text { el } \\
\text { ळ } \\
\text { establecimi } \\
\text { ento de } \\
\text { estándares }\end{array}$ \\
\hline
\end{tabular}

Autor: Reyes et al., (2017)

Elaborado por: Autores

Este tipo de modelo permitirá a los centros educativos iniciar un proceso de regulación del funcionamiento organizacional afín de satisfacer las necesidades y expectativas de los profesores, estudiantes, personal administrativo y padres de familias, así como de la competencia para mejorar de forma permanente la calidad del servicio que prestan.

La aplicación de este modelo puede mejorar el ambiente de trabajo, eliminar despilfarros, organizar los recursos, reducir pérdidas de la calidad y mejorar el cumplimiento de los estándares en materia educativa. No obstante, es un modelo con poca flexibilidad en los procesos, tiende a crear monotonía en el trabajo, ocasiona dificultad de propagación en las áreas de la organización y produce un reajuste de equipo y manejo de materiales y personal.

Mantenimiento productivo total, (TPM)

Respecto al modelo de mantenimiento productivo total (TPM), Apaza, (2015), explica que "es un enfoque gerencial que se centra en la participación de todos los empleados de una organización en la mejora del equipo" (p.17). El significado de sus siglas (TPM) en idioma inglés se resumen en la Figura No. 4. 
Figura 4. Significado del modelo TPM.

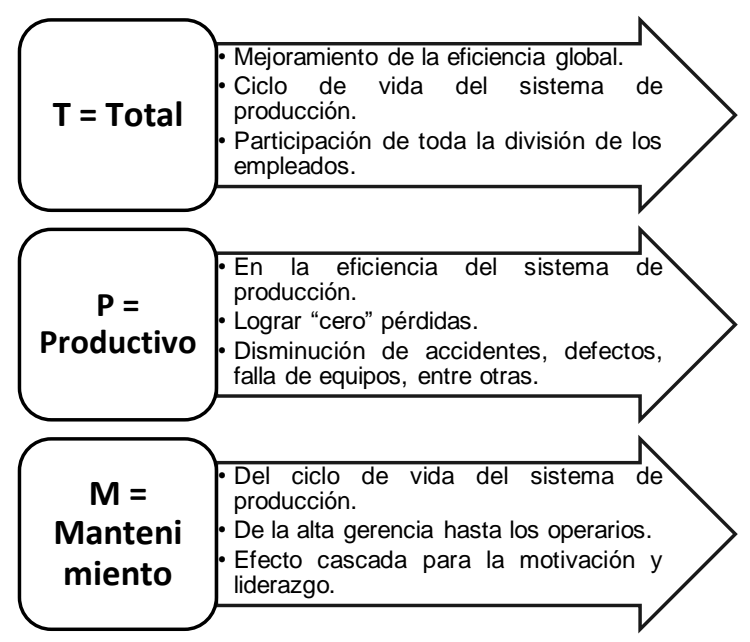

Fuente: Apaza, (2015)

Elaborado por: Autores

Es modelo aplica un método enfocado a lograr la eficiencia de equipos por medio de la participación total de los empleados, como principio básico, requiere un arduo trabajo entre todos los asociados y dueños de los procesos como la enseñanza, participación y planificación que están enfocados en la mejora continua, se orienta en el aumento de la eficiencia productiva, disminuyendo imprevistos, desperdicios, fallos y repetición de trabajos, es decir, se encamina hacia el mantenimiento proactivo e incrementa la capacidad de planificación de las actividades a desarrollarse en la organización educativa sobre cada uno de sus procesos proporcionando mayor capacidad para gestionar la calidad al interior de la entidad.
Ciclo Plan, Do, Check, Act (PDCA)

Otro modelo de gestión de la calidad es el Ciclo PDCA por sus siglas en idioma ingles: Plan, Do, Check, Act., que según, González, (2016) indica que "es conocido como el ciclo de la mejora continua porque es tan poderoso para incrementar los rendimientos de toda organización y se compone de cuatro etapas principales: planificar, hacer, verificar y actuar" (p.31).

Figura 5. Ciclo Plan, Do, Check, Act (PDCA)

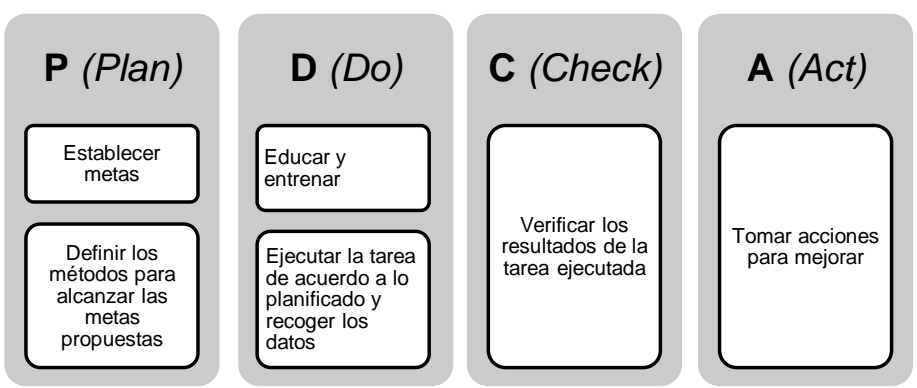

Fuente: González, (2016)

Elaborado por: Autores

Tal como se muestra en la figura No. 5. El ciclo PDCA es un modelo de gestión de la calidad que puede ser aplicado en todo tipo de organización, proceso, actividades y productos o servicios; en este sentido la etapa $P$ (plan) 0 "planificación" muestra en primer plano lo que ya se hizo para establecer lo que se logrará y así determinar las especificaciones y 
tareas que se definirán; la fase $D$ (Do) o "hacer" da inicio a la construcción del ambiente de lo planificado previamente, la fase C (check) o "verificar" se basa en la comprobación del despliegue de la aplicación de las tareas ejecutadas; y la fase A (Act) o "actuar" considera los comentarios del proceso y en base a aquello se permite rediseñar los procesos futuros.

No hay duda, que este modelo, aporta eficazmente a la gestión de la calidad en las organizaciones educativas. El cumplimiento de estas etapas garantiza la eficiencia en el proceso de enseñanza-aprendizaje y en el proceso de dirección administrativa.

\section{Modelo ISO 21001:2018}

La norma ISO 21001 (2018) en su primera versión establece un modelo para la gestión de la calidad de las organizaciones educativas con once principios que abarcan desde el enfoque a los estudiantes y otros beneficiarios, liderazgo visionario, compromiso de las personas, enfoque a procesos, mejora, toma de decisiones basada en la evidencia, gestión de las relaciones, responsabilidad social, accesibilidad y equidad, conducta ética en educación, y seguridad y protección de datos; tal como se evidencia en la figura No. 6.

Figura 6. Principios de la norma ISO 21001:2018.

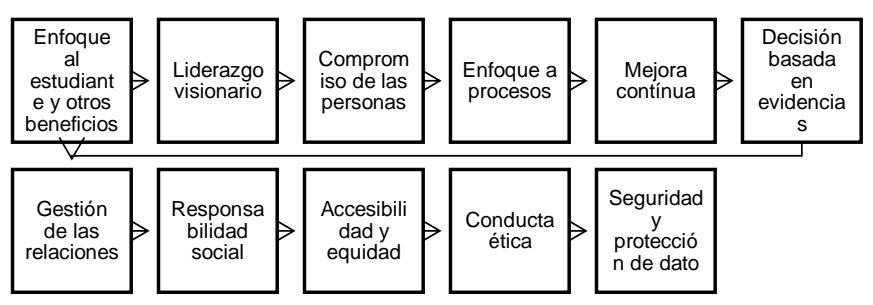

Fuente: ISO 21001:2018

Elaborado por: Autores

Los requisitos establecidos en este modelo, están fundamentados en la familia de la Norma ISO 9000 que se refiere a la gestión de la calidad en las organizaciones, no obstante, debido a la particularidad que supone la gestión dentro de las organizaciones educativas, la International Organization for Standardization (ISO) ha generado una normativa diseñada a atender las dimensiones de la gestión de la calidad desde el ámbito de la educación, dirigido a todo tipo de institución y servicio que tenga relación con la generación de conocimientos 0 procesos de enseñanza.

Sus requisitos son genéricos y aplicables a todas las 
organizaciones que utilizan un currículo para apoyar el desarrollo de la enseñanza, el aprendizaje o la investigación sin importar el tipo, tamaño o método educativo; a fin de generar preeminencias como: comunidad educativa y la sociedad en general; adecuación de los requisitos de calidad del cliente; conveniencia en las actividades y resultados del diseño del programa; aprendizaje y control del proceso de enseñanza; herramientas de marketing educativo efectivas; ventajas de acreditación nacional; potencialización de recurso humano; y mejoramiento de infraestructura y activos.

Modelo KAIZEN (Kai = cambio y Zen = bueno)

Otro modelo analizado en este estudio, aplicable en las organizaciones educativas es el modelo KAIZEN, según, Zapata, (2018) explica que: "esta metodología involucra principios y valores que soportan al sistema de gestión de la organización, de donde se establecen prácticas y herramientas de gestión, en las cuales se incluyen la Gestión por Calidad Total de la organización" (p.41). Es decir, logran el trabajo disciplinado y constante de los empleados mediante un despliegue de políticas y objetivos desde la alta dirección hasta el último empleado, tal como se muestra en la figura No.7.

Figura 7. Esfera de la filosofía gerencial de Kaizen.

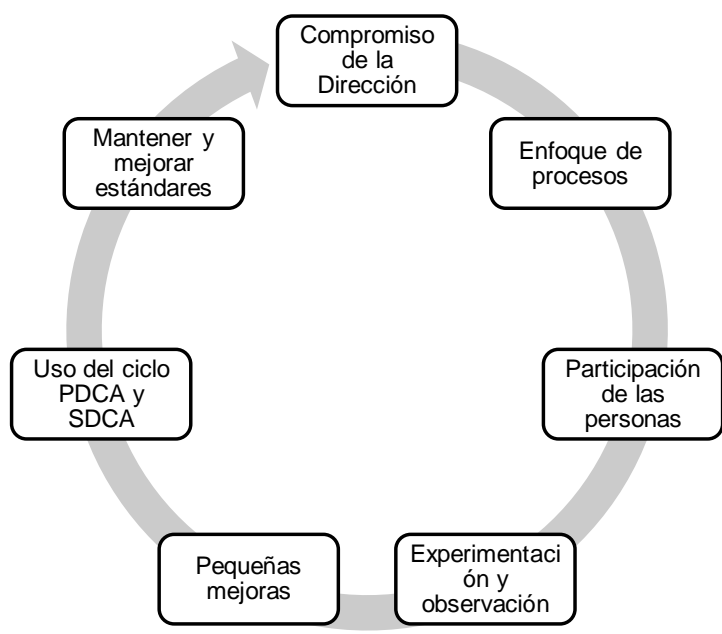

Fuente: Zapata, (2018)

Elaborado por: Autores

La metodología KAIZEN tiene su base en la teoría del comportamiento organizacional, el liderazgo transformacional, la teoría de la motivación y en enfoque de gestión por competencias que contribuye a la mejora continua del desempeño y de la calidad de la gestión educativa; este modelo recoge algunos aportes de otros modelos de gestión de la calidad, pero simplificado en pequeñas propuestas de mejora que al cabo del tiempo son significativas. 
En este sentido, para las instituciones educativas es un modelo ideal, ya que la educación es un camino que se construye día con día hasta llegar al objetivo deseado.

\section{Metodología}

El presente estudio corresponde a una tipología descriptiva de característica cualitativa, fundamentada en la revisión bibliográfica con el objetivo de analizar diferentes modelos de gestión de la calidad que se pueden implementar a la gerencia de organizaciones educativas para la mejora continua y satisfacción de las exigencias de los involucrados, es decir, estudiantes, padres de familia, profesores, directivos y demás agentes relacionados con la institución educativa.

La investigación abordó el análisis de cinco modelos de gestión de la calidad cuyas características son flexibles para ser instituidos en empresas dedicadas a ofertar servicios educativos, entre los modelos que se citan son: el modelo de las $5 \mathrm{~S}$, el modelo de mantenimiento productivo total (TPM), el modelo PDCA, el modelo ISO 21001:2018 y el modelo KAIZEN que han sido modelos adaptados en diversos países del mundo cada uno con sus características y aportes que tienen como finalidad conducir a la organización educativa hacia la gestión de cambio.

La base teórica se fundamentó en el análisis de información secundarias como: artículos científicos, secciones de libros en la web, revistas indexadas en bases científicas y normas internacionales que ayudan a comprender el funcionamiento de los modelos de gestión en los establecimientos de educación.

\section{Resultados y discusión}

En base a la revisión teórica de la presente investigación se obtuvieron los siguientes resultados:

Los modelos de gestión de la calidad analizados pueden ser instaurados en las organizaciones educativas ya que presentan características adaptables al área de los servicios, sin embargo, cada uno de ellos presentan ventajas, desventajas y particularidades de enfoque organizacional que deben ser consideradas por la entidad para tomar acciones preventivas $y$ proactivas para erradicar o minimizar las dificultades y maximizar sus 
beneficios.

En la siguiente matriz se muestran los modelos sujetos de análisis y se resaltan los aspectos que sobresalen en cada uno de ellos:

Tabla 1. Matriz comparativa de los modelos de gestión de la calidad para organizaciones educativas

\begin{tabular}{|c|c|c|c|c|}
\hline Modelo & $\begin{array}{c}\text { Significa } \\
\text { do }\end{array}$ & Ventaja & Desventaja & Enfoque \\
\hline $\begin{array}{l}\text { Las } 5 \\
\text { S: } \\
\text {-Seiri } \\
- \\
\text { Seito } \\
\mathrm{n} \\
\text { Seiso } \\
\\
\\
\text { Seiket } \\
\text { su } \\
\\
\text { - } \\
\text { Shitsu } \\
\text { ke }\end{array}$ & $\begin{array}{l}\text { Separa } \\
\text { r lo } \\
\text { innece } \\
\text { sario y } \\
\text { elimina } \\
\text { rlo. } \\
\text { - } \\
\text { Dispon } \\
\text { er y } \\
\text { ordena } \\
\text { r los } \\
\text { elemen } \\
\text { tos que } \\
\text { quedan } \\
\text { - } \\
\text { Manten } \\
\text { er } \\
\text { limpias } \\
\text { las } \\
\text { máquin } \\
\text { as y } \\
\text { ambien } \\
\text { tes de } \\
\text { trabajo. } \\
\text { - } \\
\text { Extend } \\
\text { er el } \\
\text { concep } \\
\text { to de } \\
\text { limpiez } \\
\text { a } \\
\text { - } \\
\text { Constr } \\
\text { uir } \\
\text { autodis } \\
\text { ciplina } \\
\text { median } \\
\text { ares }\end{array}$ & $\begin{array}{l}\text { - } \\
\text { el } \\
\text { ambient } \\
\text { e de } \\
\text { trabajo } \\
\text { - } \\
\text { Elimina } \\
\text { despilfa } \\
\text { rros, } \\
\text { reduce } \\
\text { pérdida } \\
\text { s de la } \\
\text { calidad. } \\
\text {-Mejora } \\
\text { el } \\
\text { cumpli } \\
\text { miento } \\
\text { de los } \\
\text { estánda } \\
\text { res. }\end{array}$ & $\begin{array}{l}\text {-Poca } \\
\text { flexibilida } \\
\text { d en el } \\
\text { proceso. } \\
\text {-Crea } \\
\text { monotoní } \\
\text { a en el } \\
\text { trabajo. } \\
\text {-Crea } \\
\text { dificultad } \\
\text { de } \\
\text { propagac } \\
\text { ión. } \\
\text { - } \\
\text { Reajuste } \\
\text { de } \\
\text { equipo y } \\
\text { manejo } \\
\text { de } \\
\text { materiale } \\
\text { s y } \\
\text { personal. }\end{array}$ & $\begin{array}{l}\text {-Inicio } \\
\text { de la } \\
\text { gestión } \\
\text { de la } \\
\text { calidad } \\
\text { en la } \\
\text { instituci } \\
\text { ón } \\
\text { educati } \\
\text { va. } \\
\text {-En el } \\
\text { funcion } \\
\text { amiento } \\
\text { y } \\
\text { desemp } \\
\text { eño del } \\
\text { trabajo. }\end{array}$ \\
\hline TPM & $\begin{array}{l}\text { Manten } \\
\text { imiento } \\
\text { product } \\
\text { ivo } \\
\text { total. }\end{array}$ & $\begin{array}{l}\text { - } \\
\text { oument } \\
\text { o de la } \\
\text { producti } \\
\text { vidad } \\
\text { laboral. } \\
\text { - } \\
\text { Reducc } \\
\text { ión de } \\
\text { fallas, } \\
\text { costes, } \\
\text { tiempo } \\
\text { y }\end{array}$ & $\begin{array}{l}\text { - } \\
\text { un } \\
\text { cambiosita } \\
\text { de } \\
\text { cultura } \\
\text { organiza } \\
\text { cional. } \\
\text {-El } \\
\text { proceso } \\
\text { de } \\
\text { impleme } \\
\text { ntación }\end{array}$ & $\begin{array}{l}\text {-Se } \\
\text { centra } \\
\text { en la } \\
\text { mejora } \\
\text { del } \\
\text { equipo. } \\
\text { - } \\
\text { Trabajo } \\
\text { por } \\
\text { compon } \\
\text { entes. } \\
\text { - } \\
\text { Planific }\end{array}$ \\
\hline
\end{tabular}

\begin{tabular}{|c|c|c|c|c|}
\hline & & $\begin{array}{l}\text { acciden } \\
\text { tes. }\end{array}$ & $\begin{array}{l}\text { requiere } \\
\text { de varios } \\
\text { años. }\end{array}$ & $\begin{array}{l}\text { ación } \\
\text { de } \\
\text { mejoras } \\
. \\
- \\
\text { Selecci } \\
\text { ón de } \\
\text { herrami } \\
\text { entas } \\
\text { adecua } \\
\text { das. }\end{array}$ \\
\hline $\begin{array}{l}\text { Ciclo } \\
\text { PDC } \\
\text { A }\end{array}$ & $\begin{array}{l}\text { Plan } \\
\text { Do } \\
\text { Check } \\
\text { Act }\end{array}$ & $\begin{array}{l}\text {-Se } \\
\text { concent } \\
\text { ra el } \\
\text { esfuerz } \\
\text { o en } \\
\text { ámbitos } \\
\text { organiz } \\
\text { ativos y } \\
\text { de } \\
\text { procedi } \\
\text { mientos } \\
\text { puntual } \\
\text { es. } \\
\text {-Dirige } \\
\text { a la } \\
\text { organiz } \\
\text { ación } \\
\text { hacia la } \\
\text { competi } \\
\text { tividad. } \\
\text { - } \\
\text { Contrib } \\
\text { uye } \\
\text { hacia el } \\
\text { avance } \\
\text { tecnoló } \\
\text { gico. }\end{array}$ & $\begin{array}{l}\text {-Se } \\
\text { pierde la } \\
\text { perspecti } \\
\text { va de la } \\
\text { interdepe } \\
\text { ndencia. } \\
- \\
\text { Requiere } \\
\text { de la } \\
\text { participa } \\
\text { ción de } \\
\text { todos los } \\
\text { integrant } \\
\text { es de la } \\
\text { organiza } \\
\text { ción y a } \\
\text { todo } \\
\text { nivel. } \\
\text { - } \\
\text { Requiere } \\
\text { important } \\
\text { es } \\
\text { inversion } \\
\text { es. }\end{array}$ & $\begin{array}{l}\text { En la } \\
\text { calidad } \\
\text { de los } \\
\text { proceso } \\
\text { s }\end{array}$ \\
\hline $\begin{array}{l}\text { ISO } \\
21001 \\
: 2018\end{array}$ & $\begin{array}{l}\text { Sistem } \\
\text { as de } \\
\text { Gestió } \\
\text { n para } \\
\text { Centro } \\
\text { s } \\
\text { Educati } \\
\text { vos }\end{array}$ & $\begin{array}{l}\text { Contrib } \\
\text { uye a la } \\
\text { mejora } \\
\text { de los } \\
\text { resultad } \\
\text { os de } \\
\text { aprendi } \\
\text { zaje. } \\
- \\
\text { Permitir } \\
\text { á } \\
\text { monitor } \\
\text { ear, } \\
\text { medir, } \\
\text { evaluar } \\
\text { y } \\
\text { analizar } \\
\text { y } \\
\text { mejorar } \\
\text { el } \\
\text { desemp } \\
\text { eño de } \\
\text { su } \\
\text { organiz } \\
\text { ación } \\
\text { educati } \\
\text { va y su } \\
\text { eficacia } \\
\text { - } \\
\text { Aplicabl } \\
\text { e a } \\
\text { todo } \\
\text { tipo y } \\
\text { tamaño } \\
\text { de }\end{array}$ & $\begin{array}{l}\text { Alto } \\
\text { costo } \\
\text { para } \\
\text { implantar } \\
\text { un } \\
\text { sistema. } \\
\text { Inversión } \\
\text { de } \\
\text { tiempo } \\
\text { en el } \\
\text { papeleo } \\
\text { Costo de } \\
\text { manteni } \\
\text { miento } \\
\text { de la } \\
\text { norma. }\end{array}$ & $\begin{array}{l}\text {-Se } \\
\text { centra } \\
\text { en la } \\
\text { alta } \\
\text { direcció } \\
\text { n, sus } \\
\text { proceso } \\
\text { s y } \\
\text { activida } \\
\text { des. }\end{array}$ \\
\hline
\end{tabular}




\begin{tabular}{|c|c|c|c|c|}
\hline & & $\begin{array}{l}\text { instituci } \\
\text { ón } \\
\text { educati } \\
\text { va. }\end{array}$ & & \\
\hline $\begin{array}{l}\text { KAIZ } \\
\text { EN }\end{array}$ & $\begin{array}{l}\text { Kai = } \\
\text { cambio } \\
\text { Zen = } \\
\text { bueno }\end{array}$ & $\begin{array}{l}\text { Reducc } \\
\text { ión de } \\
\text { fallas } \\
\text { en los } \\
\text { equipos } \\
\text { - } \\
\text { Aument } \\
\text { o en los } \\
\text { niveles } \\
\text { de } \\
\text { satisfac } \\
\text { ción de } \\
\text { los } \\
\text { clientes } \\
\text {-Mejora } \\
\text { el } \\
\text { autoesti } \\
\text { ma y } \\
\text { motivac } \\
\text { ión del } \\
\text { person } \\
\text { al. }\end{array}$ & $\begin{array}{l}\text {-Se } \\
\text { pierde la } \\
\text { perspecti } \\
\text { va de la } \\
\text { interdepe } \\
\text { ndencia. } \\
- \\
\text { Requiere } \\
\text { de un } \\
\text { cambio } \\
\text { en toda } \\
\text { la } \\
\text { organiza } \\
\text { ción. } \\
\text {-El } \\
\text { mejorami } \\
\text { ento } \\
\text { continuo } \\
\text { se hace } \\
\text { un } \\
\text { proceso } \\
\text { muy } \\
\text { largo. }\end{array}$ & $\begin{array}{l}\text { Se } \\
\text { basa en } \\
\text { identific } \\
\text { ar y } \\
\text { excluir } \\
\text { activida } \\
\text { des que } \\
\text { no } \\
\text { agrega } \\
\text { n valor } \\
\text { a la } \\
\text { empres } \\
\text { a. }\end{array}$ \\
\hline
\end{tabular}

Fuente: Apaza (2015); González (2016); Reyes et al., (2017); ISO 21001 (2018) y Zapata (2018)

Elaborado por: Autores

En efecto, los modelos de gestión de la calidad esquematizados en la Tabla No. 1 se vuelven herramientas útiles e importantes para fortalecer la planeación estratégica en la gestión interna y externa de la entidad con el contexto social.

Es importante resaltar las bondades que brindan cada uno de los modelos descritos, así el Modelo de las $5 S$ se enfoca en mantener una mejor organización, lugares más ordenados y limpios de forma permanente con el fin de incrementar la productividad y el entorno laboral; esta práctica de calidad es sencilla y efectiva, ideal para iniciar la gestión de la calidad en cualquier tipo de organización.

El modelo de Mantenimiento Productivo Total (PTM) se basa en la identificación de las pérdidas de los procesos para maximizar el uso de los activos de la entidad a fin de garantizar una obtención de servicios de calidad, este modelo es ideal para implementarse cuando una organización considere que sus requerimientos sean los de mantener plantas, equipos e instalaciones de todo tipo confiables, continuas y seguras en todos los niveles de la organización y sobre cada uno de sus colaboradores.

El modelo PDCA, conocido como el ciclo de Deming 0 de mejora continua se basa en cuatro etapas: planificar, hacer, verificar y actuar, este modelo no tiene fin, de ahí su importancia para conducir hacia la mejora continua de la organización ya que todo proceso al cumplir estas etapas obtiene como resultado un mejoramiento en la calidad, este modelo genera un contraste entre lo planificado y lo real, de acuerdo con la estandarización de cada uno de los procesos. 
El modelo ISO 21001:2018 está basado en los requisitos y principios de la ISO 9000, la cual está basada en la gestión de la calidad de todo tipo de organizaciones, sin embargo en el caso de las organizaciones educativas es necesario contar con aportes más específicos que direccionen especialmente la gestión educativa de acuerdo a los estándares internacionales de la calidad, por tanto, esta norma cubre todo el ciclo educativo, incluye principios de calidad fundamentados en la transparencia o respeto por el aprendizaje del estudiante y destaca los destinatarios de los productos y servicios de estas organizaciones a fin de estimular la excelencia y la innovación.

El modelo KAIZEN está basado en un pensamiento de mejora de manera progresiva el cual involucra a todo el personal, orienta al cliente, controla la calidad de los procesos, la automatización de las áreas directivas, la disciplina en el lugar de trabajo a fin de generar ese cambio positivo que necesita la organización para satisfacer a sus clientes internos y externos, a su vez garantiza la competitividad institucional con eficacia y eficiencia en los resultados esperados del servicio educativo existente o en el diseño de nuevos servicios educativos.

\section{Conclusiones}

Los modelos de gestión de la calidad para instituciones educativas ofrecen oportunidades para dialogar, compartir observaciones, y construir el trabajo colectivo entre actores educativos, siendo de gran utilidad para modificar las formas de ver y entender lo que acontece en el quehacer cotidiano a través de una evaluación institucional heterogénea permanente de los procesos a fin de monitorear la satisfacción de sus clientes internos y externos y tomar los correctivos oportunos.

El establecimiento de uno de los modelos de gestión de la calidad a la gestión de una organización educativa constituye una herramienta meritoria para garantizar el desarrollo sostenible de la institución, ya que están enfocados tanto en las personas como en las actividades que ejecutan y los suministros que emplean para ofertar el servicio educativo, lo que coadyuva a mejorar las salidas del servicio pedagógico. 
De acuerdo al análisis de los modelos de gestión de la calidad, se evidencia que el modelo ISO 21001:2018 es un modelo de la calidad efectivo aplicable en las instituciones educativas, ya que sus requisitos y principios están enfocados en la gestión de la calidad en organizaciones educativas que complementan la mejora de la provisión de servicios de aprendizaje, dirigido a todo tipo de institución educativa, ya sean públicas o privadas, grandes 0 pequeñas, que se encuentran relacionadas con la enseñanza, en todos los niveles, con la finalidad de dar respuestas a las exigencias de la sociedad actual, donde la educación juega un rol preponderante.

\section{Bibliografía}

Apaza, R. (2015). El modelo de mantenimiento productivo total TPM y su influencia en la productividad de la empresa minera Chama Perú E.I.R.L. Ananea-2015 [Universidad Andina Néstor Cáceres Velásquez]. PDF. http://repositorio.uancv.edu.p e/bitstream/handle/UANCV/4 38/TESIS. pdf?sequence $=3 \& \mathrm{i}$ sAllowed $=y$

Ardila, J., Ardila, M., Rodríguez, D., \& Hincapié, D. (2016). La gerencia del mantenimiento:
Una revisión. 14 (2). PDF. http://dx.doi.org/10.15665/rde .v14i2.480

De la Rosa, A., \& Guzmán, A. (2016). Profesionalización

pedagógica de los profesores universitarios:

Caso Universidad Laica Eloy Alfaro de Manabí, extensión Bahía de Caráquez. IV, 27-37.

Goairín, J., \& Díaz, A. (2016). Sistema de evaluación institucional en enseñanza obligatoria en Iberoamérica. EDO - UAB - Visión Consultores Ltda. https://ddd.uab.cat/pub/libres /2016/169885/RedAGE_siste ma_evaluacion.pdf

González, J. (2016). Metodología para fortalecer la participación del personal en el Sistema de Gestión de Seguridad y Salud en el Trabajo en una empresa del sector aeronáutico. https://doi.org/10.15332/s214 5-1389.2016.0001.05

Guerrón, J. (2019). Proyecto de dirección, empleando un cuadro de mando integral como sistema de gestión [Universidad Internacional del Rioja].

PDF. https://reunir.unir.net/handle/1 23456789/8245

ISO 21001. (2018). Educational organizations-Management systems for educational organizations-Requirements with guidance for use. 
https://www.iso.org/obp/ui\#iso :std:iso:21001:ed-1:v1:es

Ortiz, W. (2018). La gestión de calidad en el aula universitaria. $19, \quad 57-70$. https://doi.org/10.13140/RG.2 .2 .10245 .24800

Radic, J. (2017). Sistema de evaluación y mejora de la calidad educativa [Universidad Autónoma de Madrid].

https://repositorio.uam.es/bits tream/handle/10486/680578/r adic_henrici_jorge_antonio.p $\mathrm{df}$ ? sequence $=1$

Reyes, J., Aguilar, L., Hernández, J., Mejías, A., \& Piñero, A. (2017). La Metodología 5S como estrategia para la mejora continua en industrias del Ecuador y su impacto en la Seguridad y Salud Laboral. 2, 1040-1059.

https://doi.org/10.23857/pc.v2 i7.329

Ropa, B. (2014). Administración de la calidad en los servicios educativos.

4.

https://www.redalyc.org/articu lo.oa? id=570960877010

Rubia, F. (2016). Fórum Europeo de Administradores de la Educación de Aragón. 17. http://feae.eu/wpcontent/uploads/2014/05/For um-Arag\%C3\%B3n-17.pdf

Sandoval, L. (2017). Reflexiones sobre la gratuidad de la educación en el Ecuador. 21, 687-693. PDF.
Zapata, G. (2018). Modelo de gestión organizacional basado en la teoría del Kaizen para mejorar el desempeño docente en la Institución Educativa $N^{\circ} 10828$ Chiclayo [Universidad César Vallejo]. http://repositorio.ucv.edu.pe/b itstream/handle/UCV/34521/z apata_gg.pdf?sequence $=1$ \&is Allowed $=y$ 